International Journal of Biomedicine I June 2019 - Volume 9, Issue Suppl_1: Abstracts From the Second Russian International Conference "Cryo-electron microscopy 2019: achievements and prospects"

POSTER ABSTRACT PRESENTATIONS

SESSION TITLE: STRUCTURE OF MEMBRANE PROTEINS

DOI: 10.21103/IJBM.9.Suppl_1.P12

\title{
Abstract P-12: Structure of RyR1 in Native Membranes
}

\author{
Wenbo Chen $^{1,2}$, Mikhail Kudryashev ${ }^{1,2}$ \\ ${ }^{1}$ Max Planck Institute for Biophysics, Frankfurt am Main, Germany; ${ }^{2}$ Buchmann Institute for Molecular \\ Life Sciences, Goethe University Frankfurt, Frankfurt am Main, Germany
}

Background: Ryanodine receptor 1 (RyR1) mediates excitation-contraction coupling by releasing $\mathrm{Ca}^{2+}$ from the lumen of sarcoplasmic reticulum (SR) to the cytoplasm of skeletal muscle cells. RyR1 activity regulation is complicated and modulated by numerous molecules, including several regulatory proteins from both the cytoplasmic and lumenal sides of the SR, and cation ions such as $\mathrm{Ca}^{2+}$ and $\mathrm{Mg}^{2+}$, and chemicals such as caffeine and ryanodine. The activity regulation mechanism of RyR 1 is not fully elucidated, though high resolution structures of purified RyR1 in detergent solved by single particle cryoEM have been reported.

Methods: We isolated the SR from rabbit skeletal muscle and imaged RyR1 in native SR membrane with cryo-electron tomography. The structures of RyR1 in closed and open states were solved by sub-tomogram averaging, respectively.

Results: Compared to the reported structures of purified RyR1, our structure reveals the occupied competitive binding site of the regulatory proteins calmodulin and S100A1 on RyR1, helix-like densities traversing the bilayer approximately $5 \mathrm{~nm}$ from the RyR1 which are speculated to be triadin or junctin, and sarcoplasmic extensions linking RyR1 to the putative calsequestrin network. We document the major conformation or RyR1 in situ and its structural variations. Activation of RyR1 leads to significant changes of membrane curvature and sarcoplasmic extensions movement.

Conclusion: Our structures of RyR1 in situ show novel densities in cytoplasmic region and transmembrane region and SR lumen, respectively. Activation of RyR1 in situ reveals new conformational changes in the SR membrane and SR lumen. The results provide structural insights for mechanistic understanding of RyR1 in native environment.

Key Words: ryanodine receptor $\bullet$ sarcoplasmic reticulum • in situ $\bullet$ sub-tomogram averaging 
Sources of Funding: This work was supported by the Sofja Kovalevskaja Award from the Alexander von Humboldt Foundation to Mikhail Kudryashev and by a fellowship from the China Scholarship Council to Wenbo Chen

International Journal of Biomedicine. 2019;9 Suppl 1: S21-22. doi: 10.21103/IJBM.9.Suppl_1.P12 (C)2019 International Medical Research and Development Corporation 